\title{
Facial emotion recognition impairments in bipolar disorder. A cognitive problem?
}

To the editor,

In their recent study investigating emotion recognition impairments in bipolar disorder (BD), Robinson et al. (2015) report that two well-characterized groups of BD individuals failed to show performance deficits on a series of facial emotion processing tasks when compared to controls. This is in contrast to our findings of BD patient impairments on a similar set of facial emotion recognition measures in a group of stable outpatients in mixed mood states but with relatively minimal mean symptom severity (Van Rheenen \& Rossell, 2014).

Robinson et al state that methodological issues related to neurocognitive ability may account for some inconsistencies in the literature. In particular, they highlight that tasks with time pressured response formats or elevated memory demands could elicit group differences more readily than tasks without such features. We agree with this argument and speculate that it is possible that the participants in our sample were placed under greater neuropsychological pressure than those of Robinson et al., since the task used in our study did not display visual labels for each of the emotional expressions and enforced a strict inter-stimulus response window of $1500 \mathrm{~ms}$. It is arguable that these factors imposed a heightened reliance on processing speed and working memory skills.

In a previous study using a regression-based technique (path analysis) in our sample, we were able to show that a collapsed neurocognitive score encompassing processing speed, working memory, attention, learning and executive functioning measures was predictive of emotion processing in BD (Van Rheenen, Meyer, \& Rossell, 2014). This approach was useful in identifying the proportion of variance in facial emotion recognition performance that was accounted for by neurocognitive functioning in the BD group as a whole. However, recent work suggests that there is substantial 
heterogeneity in cognitive ability in individuals with this disorder (Burdick et al., 2014), with 30$40 \%$ of BD patients reported to be neuropsychologically intact (Martino et al., 2014; Martino et al., 2008). Thus, although we were able to establish the presence of a predictive relationship between neurocognition and emotion processing in our previous path analysis, we were not able to explicitly clarify whether emotion-recognition impairment was a feature shared by those BD individuals with and without neurocognitive impairment themselves.

In an attempt to place the results of both our own emotion processing study and that of Robinson et al.'s, in the context of methodological considerations related to neuropsychological demands, we conducted an exploratory re-analysis of our data (see Van Rheenen \& Rossell, 2014 for details). Specifically, we implemented a K-means clustering analysis on the BD sample reported in our 2014 paper, on the basis of performance on available processing speed (Trail Making Test A and Digit Symbol Coding subtests) and working memory (SS and LNS subtests) measures from the MATRICS Consensus Cognitive Battery (Nuechterlein et al., 2008). The analysis, using 10 iterations and a convergence criterion of zero, revealed two homogenous subgroups; one comprising 20 'impaired' patients that showed performance decrements on the clustering measures when compared to controls $(n=50)$, and one comprising 29 neuropsychologically 'equivalent' patients who performed comparably to controls on these measures.

When we analyzed performance on the emotion labeling task (referred to as the static emotion labeling task in our original study) most akin to that used by Robinson et $\mathrm{al}^{1}$ using these new dataderived groups in comparison to controls, we found significant group effects for both accuracy (F(2, $95)=3.26, \mathrm{p}<.05)$ and response time $(\mathrm{F}(2,96)=5.62, \mathrm{p}<.01)$ outcomes (Figure 1). Planned simple contrasts indicated that the neuropsychologically equivalent patient group did not differ from controls on error rate or latency across emotions and intensities overall ( $p$ 's >.05). On the other hand, the neuropsychologically impaired group demonstrated an overall decrement in performance

\footnotetext{
1 Using a 4(emotion: happy, sad, fear, angry) * 3 (intensity: high, medium, low) * 2(group; BD, control) repeated measures ANCOVA controlling for age
} 
on both outcomes (accuracy: $\mathrm{p} \leq .01$, latency: $\mathrm{p}<.001$ ), with follow-up t-tests (conservatively corrected at $\mathrm{p}<.01)$ showing that the response time effect was driven largely by slower responses for the labeling of happy and sad expressions at high intensity and happy expressions at low intensity. The accuracy effect appeared to be driven by increased errors for the labeling of angry and fear faces at high intensity and fear at medium intensity in the impaired patient group. Since there were no relationships between any of the emotion processing measures and symptom severity scores on the Young Mania Rating Scale (Young, Biggs, Ziegler, \& Meyer, 1978) or Montgomery Asberg Depression Rating Scale (Montgomery \& Asberg, 1979) in either of the neuropsychologically derived subgroups with BD (all p's >.05), mood symptomatology is unlikely to have had an effect on these results.

Given the small samples size after clustering, these findings are very preliminary and should be viewed with caution. Nonetheless they do speak to the issue raised by Robinson et al. in regards to the impact of neuropsychological impairment on emotion recognition ability. Indeed, these results in combination with that of our previous path analysis lend support to the contention that methodological differences in the extent to which facial emotion recognition tasks tap into neuropsychological processes associated with processing speed and working memory across studies, may partly explain discrepancies in the literature. This contention fits well with current neuroimaging findings of a relatively consistent, state-independent abnormality in the ventral prefrontal cortex during explicit emotion labeling in $\mathrm{BD}$; this neural region is well-known for its involvement in cognitive appraisal and re-appraisal processes as well as maintenance related working memory (D'Esposito, Postle, Ballard, \& Lease, 1999; D'Esposito, Postle, \& Rypma, 2000; Townsend \& Altshuler, 2012). In contrast, less reliably replicated abnormalities are seen in the amygdala during such tasks in $\mathrm{BD}$, with this region being typically associated with rapid automatic arousal associated with emotion detection, rather than conscious emotion recognition itself (Nomura et al., 2004; Townsend \& Altshuler, 2012). 
Taken together, it appears that emotion recognition abnormalities in $\mathrm{BD}$ might represent a problem weighted heavily by cognitive capacity, which is more apparent at the level of functional brain imaging compared to the more convoluted level of behaviour. Hence it is possible that those behavioural tasks that tap into cognition more profoundly are the ones more likely to elicit casecontrol differences in a subset of patients with BD. In light of this, we advocate that researchers assessing emotion recognition behaviorally in this disorder carefully consider the influence of neurocognitive ability in their future experimental designs.

Tamsyn Van Rheenen*a,b,c and Susan Rossell ${ }^{\text {b,c,d }}$

\footnotetext{
${ }^{a}$ Melbourne Neuropsychiatry Centre, Department of Psychiatry, University of Melbourne, Melbourne, Australia

${ }^{\mathrm{b}}$ Brain and Psychological Sciences Research Centre (BPsyC), Faculty of Health, Arts and Design, School of Health Sciences, Swinburne University, Melbourne, Australia

${ }^{\mathrm{c}}$ Cognitive Neuropsychiatry Laboratory, Monash Alfred Psychiatry Research Centre (MAPrc), The Alfred Hospital and Central Clinical School, Monash University, Melbourne, Australia.

${ }^{\mathrm{d}}$ Department of Psychiatry, St Vincent's Hospital, Melbourne, Australia
} 


\section{References}

Burdick, K. E., Russo, M., Frangou, S., Mahon, K., Braga, R. J., Shanahan, M., \& Malhotra, A. K. (2014). Empirical evidence for discrete neurocognitive subgroups in bipolar disorder: clinical implications. Psychological Medicine, 44(14), 3083-3096. doi: http://dx.doi.org/10.1017/S0033291714000439

D'Esposito, M., Postle, B. R., Ballard, D., \& Lease, J. (1999). Maintenance versus Manipulation of Information Held in Working Memory: An Event-Related fMRI Study. Brain and Cognition, 41(1), 66-86. doi: http://dx.doi.org/10.1006/brcg.1999.1096

D'Esposito, Mark, Postle, Bradley R, \& Rypma, Bart. (2000). Prefrontal cortical contributions to working memory: evidence from event-related fMRI studies. Experimental brain research, 133(1), 3-11.

Martino, Diego J, Strejilevich, Sergio A, Marengo, Eliana, Ibañez, Agustín, Scápola, María, \& Igoa, Ana. (2014). Toward the identification of neurocognitive subtypes in euthymic patients with bipolar disorder. Journal of affective disorders, 167, 118-124.

Martino, Diego J., Strejilevich, Sergio A., Scápola, María, Igoa, Ana, Marengo, Eliana, Ais, Ezequiel D., \& Perinot, Lila. (2008). Heterogeneity in cognitive functioning among patients with bipolar disorder. Journal of Affective Disorders, 109(1-2), 149-156. doi: 10.1016/j.jad.2007.12.232

Montgomery, S.A. , \& Asberg, M. (1979). A new depression scale designed to be sensitive to change. British Journal of Psychiatry, 134, 382-389.

Nomura, Michio, Ohira, Hideki, Haneda, Kaoruko, Iidaka, Tetsuya, Sadato, Norihiro, Okada, Tomohisa, \& Yonekura, Yoshiharu. (2004). Functional association of the amygdala and ventral prefrontal cortex during cognitive evaluation of facial expressions primed by masked angry faces: an event-related fMRI study. Neuroimage, 21(1), 352-363.

Nuechterlein, Keith H., Green, Michael F., Kern, Robert S., Baade, Lyle E., Barch, Deanna M., Cohen, Jonathan D., . . Marder, Stephen R. (2008). The MATRICS Consensus Cognitive Battery, part 1: test selection, reliability, and validity. The American journal of psychiatry, 165(2), 203.

Robinson, Lucy J., Gray, John M., Burt, Mike, Ferrier, I. Nicol, \& Gallagher, Peter. (2015). Processing of Facial Emotion in Bipolar Depression and Euthymia. Journal of the International Neuropsychological Society, 21(09), 709-721. doi: doi:10.1017/S1355617715000909

Townsend, Jennifer, \& Altshuler, Lori L. (2012). Emotion processing and regulation in bipolar disorder: a review. Bipolar Disorders, 14(4), 326-339. doi: 10.1111/j.13995618.2012.01021.x

Van Rheenen, Tamsyn E., Meyer, Denny, \& Rossell, Susan L. . (2014). Pathways between neurocognition, social cognition and emotion regulation in bipolar disorder. Acta Psychiatrica Scandinavica, 30(5), 397-405.

Van Rheenen, Tamsyn E., \& Rossell, Susan L. (2014). Let's face it: facial emotion processing is impaired in bipolar disorder. Journal of the International Neuropsychological Society, 20(02), 200-208. doi: 10.1017/S1355617713001367

Young, RC, Biggs, JT, Ziegler, VE, \& Meyer, DA. (1978). A rating scale for mania: reliability, validity and sensitivity. The British Journal of Psychiatry, 133(5), 429-435. doi:

10.1192/bjp.133.5.429 
a)

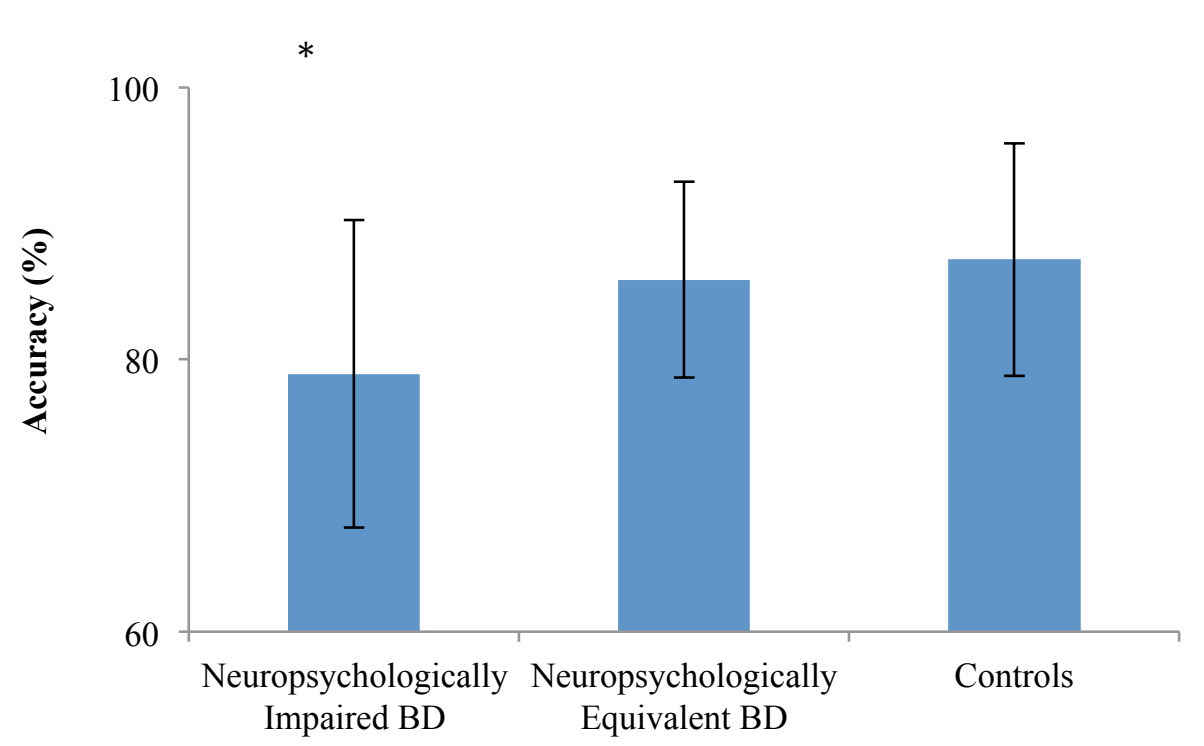

b)

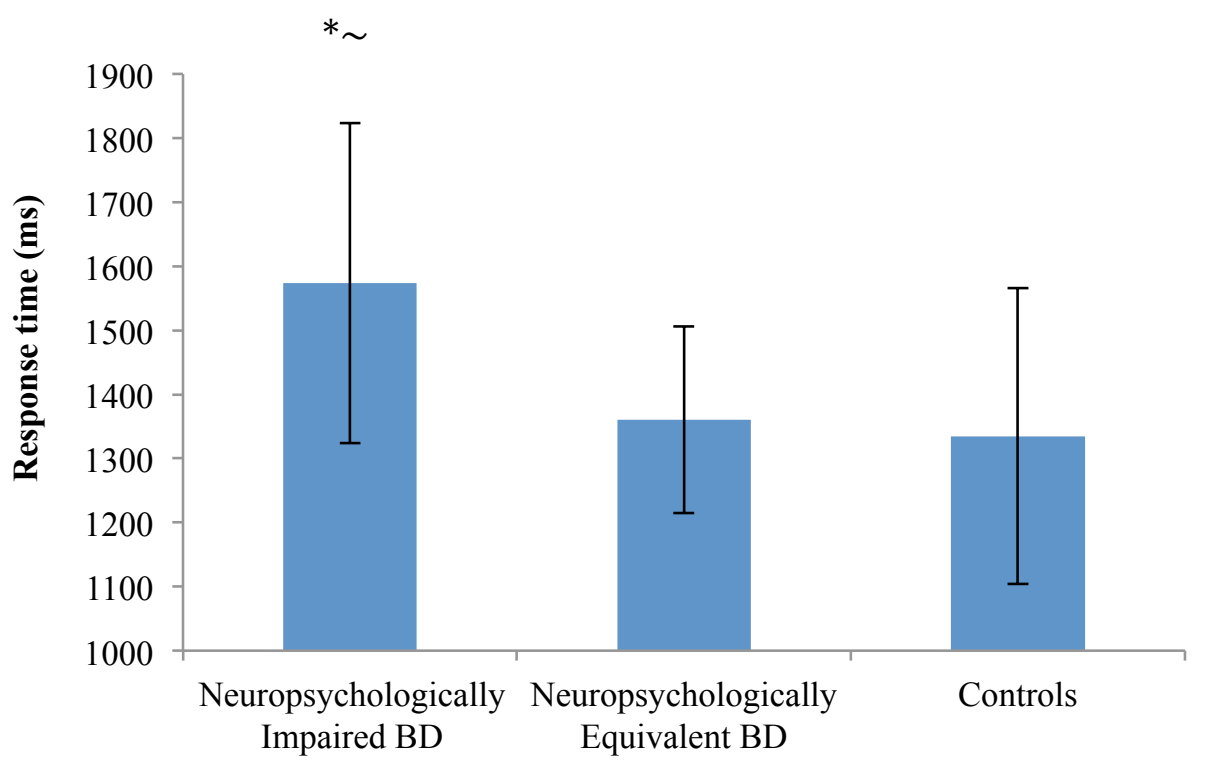

Figure 1. a) Group differences in overall accuracy performance across emotions and intensities; $b$ ) Group differences in overall response time performance across emotions and intensities. Note *neuropsychologically impaired patients compared to controls $p<.01 ; \sim$ compared to neuropsychologically equivalent patients $\mathrm{p}<.01$. Effect sizes in Cohen's $\mathrm{d}$ (accuracy/response time) between groups are as follows: neuropsychologically impaired patients compared to controls $\mathrm{d}=-0.88 ; 0.99$ and neuropsychologically equivalent patients $\mathrm{d}=-0.73 ; 1.04$; neuropsychological equivalent patients compared to controls $\mathrm{d}=-0.19 ; 0.1 .3$ 


\section{Conflicts of interest}

Dr Van Rheenen is currently supported by an NHMRC Early Career Fellowship.

\section{Acknowledgements}

The collection of data presented in this letter was made possible through the support of the Helen McPherson Smith Trust, the Australian Rotary Health/Bipolar Expedition and an Australian Postgraduate Award 